\title{
Energía y medioambiente para la habitabilidad en la ciudad histórica de Santiago de Compostela. 1994-2012
}

\author{
Ángel Panero Pardo | Oficina Técnica del Consorcio de Santiago de Compostela \\ URL de la contribución <www.iaph.es/revistaph/index.php/revistaph/article/view/4965>
}

\section{RESUMEN}

El artículo hace un recorrido sintético y subjetivo por el proceso de recuperación urbana de la ciudad histórica de Santiago de Compostela puesto en marcha tras su reconocimiento como Patrimonio de la Humanidad en 1985. En función de la complejidad de este proceso de recuperación del patrimonio en el marco de un proyecto general para la ciudad completa, que asumía simultáneamente en aquel momento la designación como capital de Galicia, el texto aborda algunas de las claves del proceso y del conocimiento adquirido durante su ejecución. Tras referir una última década del siglo XX que incorporó la consideración específica y central de las circunstancias de la vida cotidiana de los habitantes que resistían en la ciudad histórica, el texto se centra en el periodo 2006-2012, en el que se trató de construir una propuesta de recuperación urbana donde la ciudad histórica se contempla más como un ecosistema que como un monumento.

Este intento de reformular la recuperación urbana de una ciudad patrimonio de la humanidad ensayado en Santiago de Compostela, y la estimulante construcción de nuevos discursos urbanos que genera, se debe entender coherente con la Recomendación sobre Paisaje Urbano Histórico Unesco del año 2011 y con los Objetivos del Desarrollo Sostenible definidos en 2015 por la ONU. El artículo pretende evidenciar el esfuerzo por transformar el discurso tradicional de la conservación del patrimonio urbano, anclado en ocasiones en criterios ambientalistas decimonónicos, tratando de vincularlo con estrategias de conservación del cambio continuo característico de cualquier "ciudad viva".

\section{Palabras clave}

Centros históricos | Ciudades históricas | Conservación (Patrimonio) | Custodia del territorio | Eficiencia energética | Gestión administrativa | Habitabilidad | Mantenimiento | Medioambiente | Oficina Técnica del Consorcio de Santiago de Compostela | Participación ciudadana | Patrimonio urbano | Recuperación urbana|Rehabilitación arquitectónica|Revitalización | Santiago de Compostela (Galicia) | Sostenibilidad | Viviendas | 


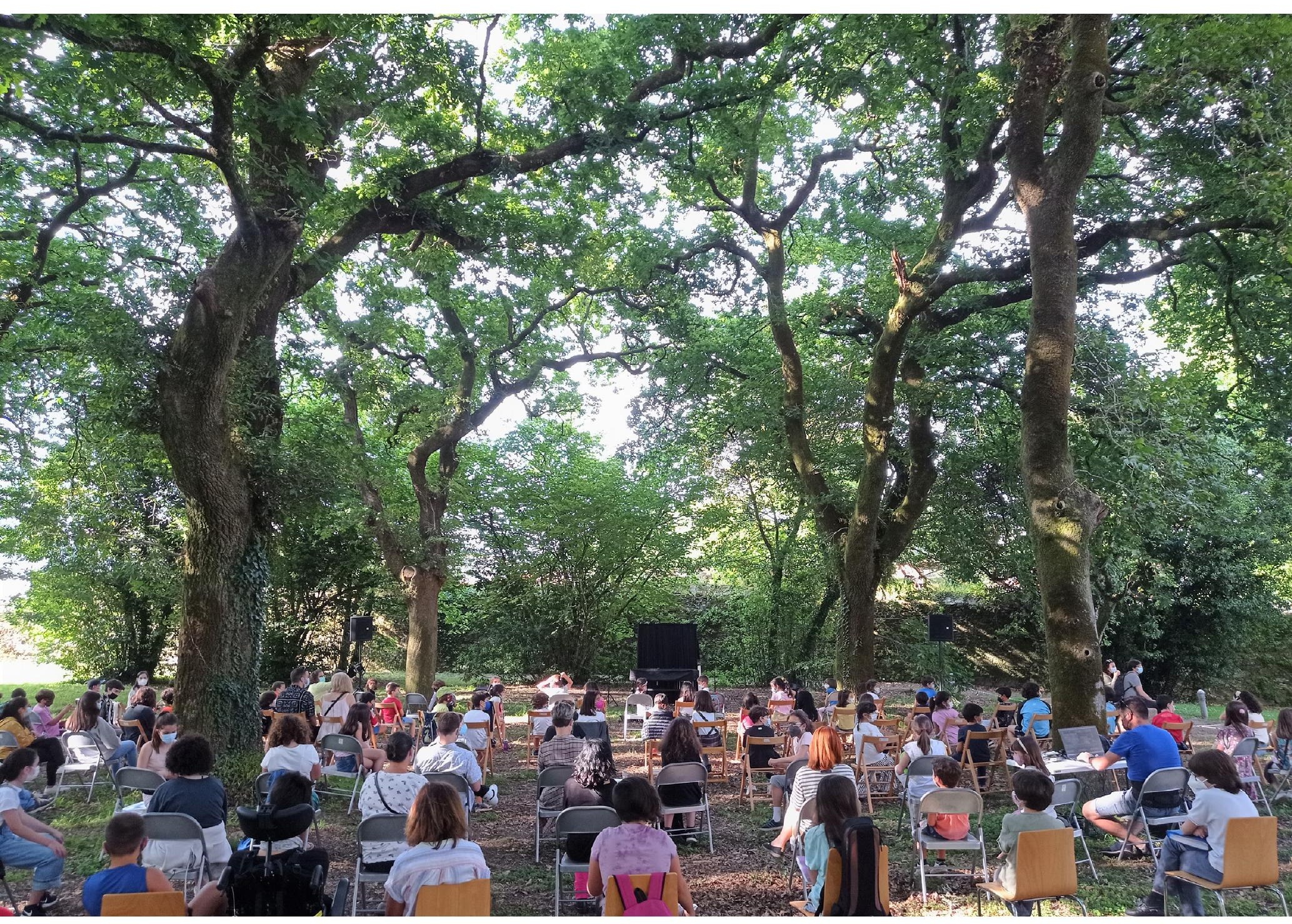

Desde el año 2006, la conservación del patrimonio urbano de Compostela se comenzó a abordar desde una perspectiva ecosistémica. La recomendación Unesco sobre paisaje urbano histórico y los ODS2030 reforzaron más tarde una línea de trabajo que hoy es ya indispensable para gestionar las consecuencias del cambio climático y los déficits urbanos que dejó al descubierto la COVID-19 | foto Ángel Panero Pardo 
Pocos días antes del comienzo del presente Año Santo 2021, se cumplieron 35 años de la incorporación de Santiago de Compostela a la lista Unesco de ciudades Patrimonio de la Humanidad. A continuación expondré una historia necesariamente subjetiva y sintética de las iniciativas desplegadas tras aquel reconocimiento, con especial atención al periodo 20062012, en el que tuve la responsabilidad de promover desde el Consorcio de Santiago el esfuerzo colectivo por formular una estrategia innovadora de actuación en la que, a los programas tradicionales de intervención, focalizados durante el siglo XX desde la perspectiva dominante de la conservación del patrimonio, se incorporaron principios propios de la, en aquel entonces incipiente, reflexión colectiva mundial contemporánea sobre ciudad, desarrollo sostenible y combate del cambio climático antropogénico. Este cambio sustancial en la forma de entender la ciudad histórica y la estrategia para garantizar su conservación en el marco de una realidad urbana compleja se había iniciado con la experiencia de intervención de la última década del siglo XX en la ciudad de Santiago, en la que cobraron importancia prioritaria la ciudadanía y las necesidades de sus vidas cotidianas, como bien ilustra el nombre No todo es fachada del primer programa de rehabilitación lanzado en 1994.

Más de una década después de aquel primer programa se comenzó a construir desde el Consorcio de Santiago una propuesta de intervención en la que la ciudad histórica se aborda, definitivamente, más como un ecosistema que como un monumento. Esta nueva perspectiva ecosistémica de la conservación del patrimonio urbano en un planeta cada vez más urbanizado se ha venido reforzando progresivamente hasta hoy, no ya por la conciencia de reacción necesaria asumida por la humanidad en su conjunto para combatir las consecuencias del cambio climático, sino hoy con todavía mayor actualidad, como consecuencia de los déficits urbanos que ha dejado al descubierto la pandemia provocada por la COVID-19 que asola el mundo desde los primeros meses del año 2020.

Aunque pueda parecer inicialmente ajena a la metodología del objetivo de conservación del patrimonio urbano tal y como se ha entendido mayoritariamente durante un siglo, en realidad, esta nueva forma de abordar los problemas de los centros históricos desde la energía, el medioambiente y los asuntos esenciales de la vida cotidiana nos sitúa ante la gran oportunidad de reintroducir en la agenda urbana de los centros históricos aspectos elementales de la gestión de las ciudades ignorados durante más de un siglo por políticos, ciudadanos, economistas, arquitectos, urbanistas y especialistas en conservación del patrimonio urbano. Y la buena noticia es que esos aspectos imprescindibles para enfrentar con garantías la recuperación del patrimonio urbano se encuentran ya, implícita y explícitamente, dentro de la recomendación de la Unesco relativa al denominado paisaje urbano histórico (Unesco 2011). 


\section{LA CIUDAD COMO ECOSISTEMA}

Creo que la experiencia en la gestión de programas de rehabilitación urbana nos ha hecho empezar a sospechar que la intervención sobre la ciudad histórica tiene más que ver con el manejo de la transformación permanente y el cambio continuo que con la estricta conservación del patrimonio. Si bien creo que aún no hemos sido capaces de superar con nitidez el lastre patrimonialista que ha destrozado la gestión efectiva de las áreas urbanas señaladas por su valor cultural, las políticas de recuperación urbana de centros históricos en los últimos 50 años parecen haber evolucionado afortunadamente desde el ensimismamiento decimonónico centrado en la conservación de la apariencia, para incorporar, primero, la atención por las arquitecturas civiles anónimas o secundarias; más tarde, cuando ya casi todos se habían ido, la atención a los asuntos de la vida cotidiana de los habitantes; $y$, finalmente, la reflexión integrada de la ciudad histórica en la ciudad completa y su territorio de vida.

En realidad, creo que este cambio sustancial en la forma de manejar el patrimonio cultural que representan las ciudades históricas ha venido inducido, en primer lugar, por el fracaso estrepitoso de las metodologías conservacionistas al uso, ineficientes y muy inadaptadas a la compleja realidad urbana de un centro histórico, y, en segundo lugar, por la transformación que en la cultura dominante ha provocado la constatación de que el cambio climático está vinculado con la actividad de los seres humanos y por tanto, en gran medida, con la forma de habitar en las ciudades. En este contexto es en el que las ciudades históricas progresivamente se han podido empezar a contemplar como ecosistemas de éxito contrastado en el tiempo para la vida en comunidad de las personas, y por tanto, y esto me parece muy importante, como modelos de referencia más que como áreas a rescatar o escenografías amables a conservar por su belleza, trascendencia o contribución a la construcción del discurso histórico-artístico oficial.

\section{CIUDAD DE LA RENTA VERSUS CIUDAD DE LA REPRODUCCIÓN SOCIAL}

Desde el respeto que merece la legislación vigente, nacida con carácter eminentemente defensivo, y más que solamente respeto, desde mi solidaridad y obediencia debida a los técnicos e incluso políticos que desarrollan su actividad al servicio de la administración en relación con la protección del patrimonio urbano, tengo la sensación de que aún hoy en demasiados casos, el enfoque oficial de aproximación a la conservación de las ciudades históricas tiene tintes de cierta ingenuidad, o quizás romanticismo naíf, que se hace muy evidente precisamente por ignorar, o no querer ver, el contexto de extraordinarias tensiones por los intereses económicos y poderes fácticos afectados en el que se desarrollan las políticas de protección. Yo reconozco ahí una palanca letal para la conservación del patrimonio urbano, que se 
produce cuando se coordinan nefastamente las legislaciones culturalmente bienintencionadas de protección del patrimonio, por ejemplo en relación con el señalamiento de catalogación, con la realidad de la expectativa de renta que con naturalidad domina cualquier movimiento en la ciudad, y con mayor razón, en la ciudad histórica, que ostenta por definición una posición de referencia y centralidad estratégica y representativa en el teatro de operaciones urbano. No me extiendo sobre este modelo vigente, económico pero también cultural, de "ciudad de la renta" frente al modelo de "ciudad de la reproducción social", porque lo mejor que recomiendo es leer al profesor Alfonso Álvarez Mora (2016) sobre el particular. Pero sí quiero insistir antes de seguir en que debemos tener siempre presente que nuestro trabajo con el objetivo de la conservación de las ciudades históricas se produce sobre suelo urbano tenso, en un contexto eminentemente expectante desde el punto de vista inmobiliario, muy asumido socialmente y organizado para producir renta y plusvalías con naturalidad. No quiero entrar aquí a opinar sobre esta circunstancia, por otro lado consustancial y coherente con el sistema económico establecido, pero sí quiero denunciar primero, que el único discurso sobre la ciudad que fuimos capaces de construir y enriquecer en la última mitad del siglo XX fue ese discurso inmobiliario neoliberal, y reivindicar después, que, más allá de la discusión ideológica, debemos emplearnos a fondo para construir colectivamente y cuanto antes otros discursos alternativos al de las plusvalías inmobiliarias, en muchos casos esenciales incluso para la supervivencia, que es en mi opinión la mejor forma de neutralizar el dominio de la "ciudad de la renta" sobre la "ciudad de la reproducción social", y encontrar razones y argumentos de peso tanto para hacer crecer la ciudad como para rehabilitar la ciudad histórica (Rueda Palenzuela 2018). Esta conciencia de actuar en suelo urbano tenso y la conveniencia de neutralizar la expectativa de renta es por tanto la primera condición previa que me gustaría apuntar para armar un proyecto de renovación urbana. Creo que el Plan Especial de Santiago supo apoyar su estrategia de recuperación en ello y eso abrió la oportunidad a que sucedieran otras cosas interesantes.

\section{COMPETENCIA MUNICIPAL, COOPERACIÓN INTERADMINISTRATIVA Y PROYECTO URBANO}

Las otras consideraciones previas que me parecen determinantes y que, a pesar de su obviedad, son sin embargo muy difíciles de encontrar en la práctica, son las siguientes:

1. Competencia municipal. Que significa que la rehabilitación de la ciudad histórica y su puesta en valor es un proyecto de competencia e iniciativa municipal que necesariamente debe implicar a la ciudad en su conjunto y formularse en el marco de la ciudad completa y su territorio de vida. La figura esencial es desde mi punto de vista el alcalde, que es quien debe liderar el 
proyecto. A pesar del asincronismo entre los tiempos de la política y los tiempos del proyecto urbano, sigo convencido de la importancia determinante de la política en los procesos de recuperación urbana de las ciudades históricas, no solo para formular y poner en marcha un proyecto de recuperación sino, y quizás más complicado, para sostenerlo en el tiempo.

2. Cooperación entre las administraciones. La complejidad y transversalidad de las cuestiones que es preciso abordar requieren de la cooperación y coordinación entre administraciones. La cooperación entre administraciones genera confianza entre la ciudadanía y permite abordar con ventaja muchas de las acciones de oportunidad que pueden tirar del proceso. El Consorcio de la ciudad de Santiago, creado en 1992 y participado por el Gobierno de España, el Gobierno de Galicia y el Gobierno municipal, ha sido, bajo la presidencia del alcalde y ejecutando los acuerdos del Real Patronato de Santiago de Compostela que preside S.M. el Rey de España, una herramienta esencial en el proceso de modernización urbana y rehabilitación de la ciudad histórica de Santiago.

3. Proyecto urbano. Frente a la tentación de impulsar la rehabilitación de la ciudad histórica a golpe de ocurrencias, que es bastante corriente, es hoy más necesario que nunca reivindicar la importancia de tener un proyecto para la ciudad completa y una estrategia para su ejecución.

Estas consideraciones previas, entre otras que se podrían añadir, se refieren a condiciones de contexto que, desde mi punto de vista, son indispensables para poner en marcha con garantías de éxito la compleja recuperación urbana de una ciudad histórica.

Las ciudades históricas han sobrevivido a situaciones aún peores en la historia y son un modelo de referencia. Yo estoy convencido de que la preservación del patrimonio urbano ya no requiere tanto de cautelas y políticas públicas defensivas, como de un amplio compromiso colectivo con el futuro en el que siguen teniendo plena vigencia y actualidad las salvaguardas de la urbanística que definió ya en 1976 el profesor Campos Venuti: pública, social, productiva, ambiental y programática (Campos Venuti 1981). Hoy en día, creo que cualquier proyecto de recuperación urbana de una ciudad histórica, además de las condiciones mínimas de contexto referidas, debe armarse incorporando coherentemente otras consideraciones relativas a la energía, el medioambiente, la política de vivienda o la formación para el empleo.

\section{LOS PRIMEROS AÑOS 1994-1999}

La etapa inicial entre 1994 y 1999 permitió consolidar bajo la dirección del arquitecto Javier Ramos Guallart una metodología de intervención basada 
El trabajo inicial de la Oficina Técnica del Consorcio, entre los años 1994 y 1999, permitió consolidar bajo la dirección de Javier Ramos Guallart una metodología de intervención basada en la atención especializada, directa y personalizada a los ciudadanos. Se pusieron en marcha más de 1.000 pequeñas intervenciones de mejora de la habitabilidad con el programa "No todo es fachada" foto Ángel Panero Pardo en la atención especializada directa y personalizada a los ciudadanos. Los técnicos de la administración se implicaban de la mano de los habitantes en la búsqueda de soluciones con el objetivo de mejorar la habitabilidad de sus viviendas, caso a caso. Yo en esta etapa le concedo gran importancia al ritmo de implementación y a una cuestión de género. La cuestión de género tiene que ver con el hecho de que normalmente las interlocutoras eran las mujeres de la casa, poseedoras de un conocimiento más preciso e inteligente de las claves de habitabilidad del hogar familiar. El ritmo de implementación de los programas tiene que ver con que se difundieron por capilaridad entre los habitantes mediante un proceso de transmisión boca a boca en el que el éxito constructivo de las pequeñas intervenciones, basadas en la coherencia tecnológica con la construcción vernácula, y las evidentes mejoras de las condiciones de habitabilidad que sin embargo se materializaban, terminaron por conquistar la confianza de los ciudadanos y demostrar que la rehabilitación pacífica del patrimonio urbano no solo era posible, sino también rentable económica y socialmente. Muchas de las intervenciones se realizaron sobre viviendas ocupadas con alquileres de renta antigua, lo que fue posible porque el porcentaje de subvención se establecía con la autorización del propietario pero en base a los ingresos de los inquilinos, a condición de que se mantuvieran en la casa tras las obras de mejora. Esto permitió establecer indirectamente un escudo de salvaguarda sobre habitantes en la tercera edad, que en muchos casos habían resistido en la ciudad histórica sencillamente porque no se podían ir a otro sitio. Se recuperaron cientos de

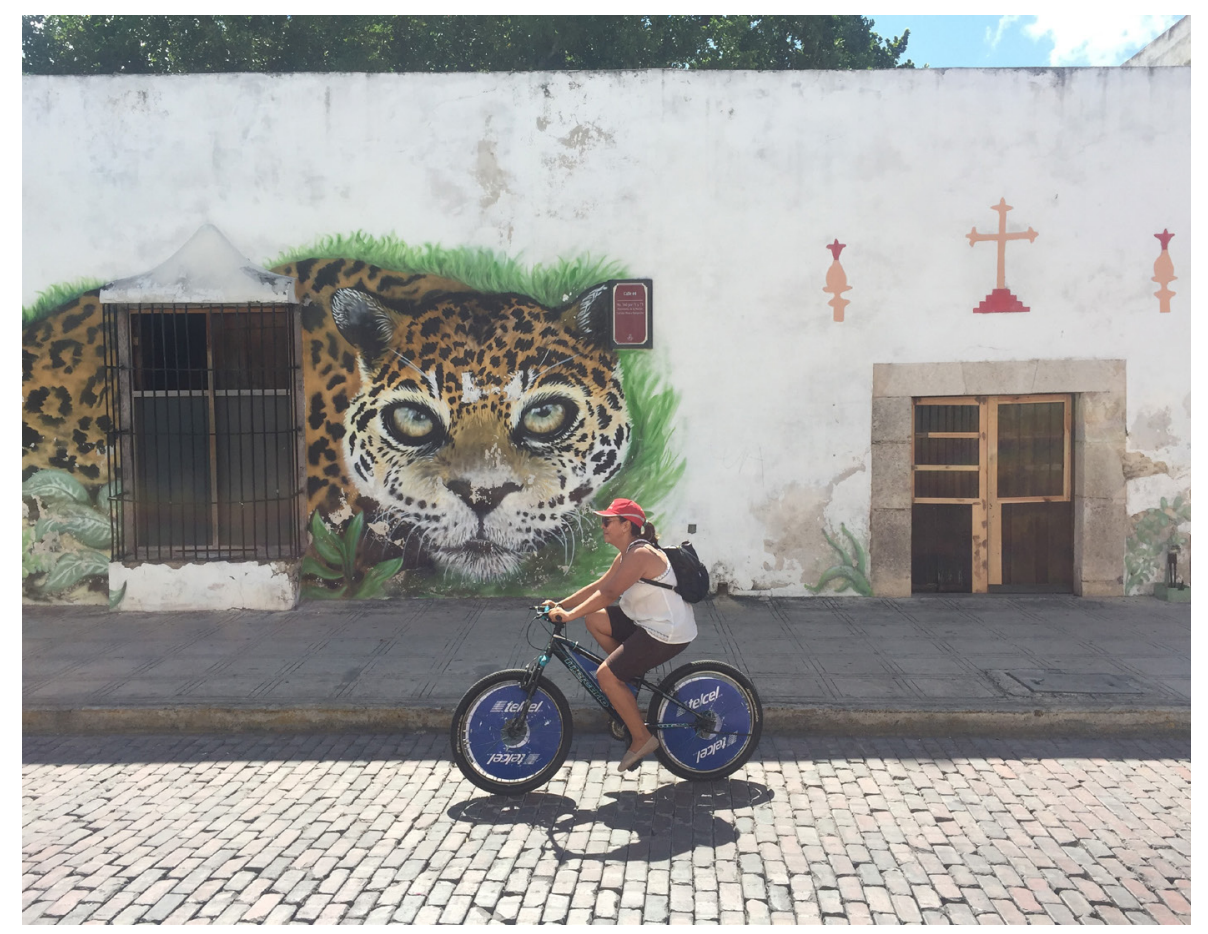


viviendas y, lo más importante, se consiguieron extraordinarios avances en la cultura constructiva coherente con las características de la arquitectura vernácula de la ciudad histórica de Santiago. El paso del tiempo matizó por ley natural el éxito de esta etapa, pues la mejora en el soporte físico de las viviendas, con la llegada de nuevos inquilinos, terminó por dejar las viviendas rehabilitadas en el rango más alto de precios de alquiler o venta de toda la ciudad.

La etapa de recuperación urbana de la ciudad histórica dirigida por Javier Ramos Guallart, en la que el trabajo de la Oficina Técnica del Consorcio recibió, entre otros, el Premio Europa Nostra 1996, el Premio Gubbio 1996, el Primer Premio Europeo de Urbanismo apartado Local Planning 1997 y el Premio Internacional Dubai de Buenas Prácticas 2002, concluyó en el año 2000 con una operación mediática de acoso y derribo, promovida, con algún que otro apoyo interno, por algunos poderes fácticos agraviados por la dinámica de recuperación urbana impulsada. La operación frenó la dinámica de rehabilitación urbana normalizada y el Consorcio de Santiago entró en una etapa de perfil muy bajo durante 5 años. Javier Ramos pasó a ser Secretario General de Vivienda del Gobierno de España entre el año 2004 y el 2010 (Ramos Guallart et ál. 2002a, 2002b).

\section{EL PERÍODO 2006-2011, ENERGÍA Y MEDIOAMBIENTE PARA IMPULSAR EL PROCESO DE RECUPERACIÓN URBANA DE LA CIUDAD HISTÓRICA DE SANTIAGO DE COMPOSTELA}

En el año 2006, tras la llegada de Xosé Manuel Villanueva a la gerencia del Consorcio, se inició el diseño de una nueva programación plurianual de iniciativas con el objetivo de reactivar el aletargado proceso de recuperación urbana de la ciudad histórica de Santiago. La experiencia acumulada en Compostela, la coyuntura global en relación con la ciudad y el territorio, la contrastada insuficiencia de las herramientas y estrategias tradicionales de conservación del patrimonio, requerían innovación desarrollando fórmulas para comprometer la responsabilidad activa de la ciudadanía y promover enfoques alternativos a los tradicionales para la conservación y puesta en valor del patrimonio urbano. Y todo ello con el convencimiento de que el patrimonio cultural urbano es ante todo expresión de la vida cotidiana de las personas y solo puede permanecer vivo en un proceso de cambio continuo y transformación permanente, lo que por otro lado explica su permanencia en el tiempo hasta nuestros días, y es por tanto garantía de su conservación.

Se puede decir que, en resumen, el espíritu central de aquella programación definida durante el año 2006 giraba en torno a la idea de introducir la energía en la agenda urbana de la renovación y revitalización de una Ciudad Patrimonio de la Humanidad. No se trataba de volver a desplegar políticas 
tradicionales de rehabilitación, que por otro lado se habían implementado con éxito como se ha dicho durante el último decenio del siglo $X X$, sino de inducir una reflexión transversal y profunda sobre el hecho urbano, reincorporando aspectos determinantes en la vida cotidiana de los habitantes y en la mejora de su calidad de vida lamentablemente olvidados en la gestión de la ciudad durante el siglo pasado. La forma en la que gestionamos el agua o la vegetación en la ciudad, el diseño de programas de habitabilidad y mantenimiento más que de rehabilitación, la reflexión sobre las infraestructuras urbanas o el uso del espacio público, más allá de su recurrente acondicionamiento epidérmico, la generación de conocimiento y empleo local asociado al proceso de recuperación urbana, la implicación activa de los ciudadanos en procesos de custodia del patrimonio urbano, o cómo los suelos fértiles agrícolas pueden ser un factor en la ordenación del suelo y la economía local, están en el código genético de todas las ciudades históricas, y no tenemos más remedio que volver a incorporarlos en la reflexión sobre la ciudad futura con la que soñamos.

Entre otras en las que no entraré por razones de espacio, quiero destacar aquí las siguientes líneas de trabajo principales:

> Gestión de la información y diligencia burocrática.

> Política de vivienda en la ciudad histórica.

$>$ Energía y medioambiente para la habitabilidad.

\section{Gestión de la información y diligencia burocrática}

La reforma de la administración pública y de la gestión de las ciudades históricas, derrocando el imperio actual de la decimonónica burocracia defensiva que ralentiza cuando no bloquea todo tipo de iniciativas, es indispensable para impulsar la senda de la revitalización de los centros históricos. También porque resulta vital aprender a manejar con precisión y precocidad la ingente información y establecer un criterio de indicadores de referencia que nos permitan conocer las consecuencias y efectos de los programas de recuperación urbana, porque muchas veces, cuando esos efectos son evidentes es demasiado tarde para hacer correcciones.

Por esta razón, en colaboración con el Instituto Geográfico Nacional, y con la colaboración del Consejo Superior de Investigaciones Científicas, el Consorcio de Santiago desarrolló el Sistema de Información Patrimonial (SIP) de la ciudad, pensado no solo como un depósito de información al servicio del conocimiento, sino como una herramienta efectiva de gestión cotidiana de la ciudad histórica. Además de la agrupación fácilmente accesible de todo tipo de información gráfica y escrita sobre el patrimonio inmue- 
ble de la ciudad histórica de Santiago, el objetivo superior era normalizar la gestión del día a día de la administración pública utilizando esta poderosa herramienta de información geográfica, única forma razonable de mantener actualizados sus contenidos. Desgraciadamente la incorporación normalizada de su uso al día a día de la gestión administrativa de las diferentes administraciones públicas implicadas está hoy en día todavía pendiente. Los tiempos y procesos de la tramitación administrativa de las licencias de obras que estamos manejando hoy en día en cualquier centro histórico del mundo son sencillamente inadmisibles, y lastran de forma irreversible cualquier dinámica de revitalización. Hay un porcentaje no menor de licencias de construcción para intervenir en la ciudad histórica que se podrían llegar a tramitar con normalidad en cuestión de minutos. Un Plan Especial vigente, el Sistema de Información Patrimonial y la colaboración de arquitectos y aparejadores a modo de fedatarios públicos creemos que lo harían posible.

\section{Política de vivienda en la ciudad histórica}

España es un país de viviendas vacías. El 13,7 \% de las viviendas de España están vacías. En Galicia, aún peor, están vacías 1 de cada 5 viviendas (Verdú 2011). Es urgente e imprescindible reorientar las políticas de rehabilitación urbana y la inversión pública en rehabilitación hacia la política de vivienda. Sobre todo, pero no solo, en los centros históricos. La cultura inmobiliaria, y la irrupción de las nuevas fórmulas de mercado asociadas a las plataformas tecnológicas, obliga a abordar sin demora con intensidad el problema de la vivienda en los ámbitos de las ciudades históricas, y con mayor razón en las ciudades patrimonio de la humanidad, que se encuentran singularmente asediadas.

El Consorcio de Santiago lanzó en 2008 un programa de rehabilitación de edificios tutelados (Bases 2008), en el que básicamente se ofrecía al propietario la rehabilitación del edificio a cambio del usufructo durante un plazo determinado gestionando las viviendas resultantes en alquiler hasta equilibrar la inversión realizada por la administración pública en la rehabilitación física del inmueble. Se llegó a realizar a través de la Empresa Municipal de la Vivienda el expediente de un inmueble con tres viviendas, cuya rehabilitación costó $279.000 €$ y el periodo de usufructo se estableció en 17 años. (Panero Pardo 2012).

\section{Energía y medioambiente para la habitabilidad}

En la búsqueda de innovación y nuevas fórmulas de aproximación urbana, uno de los objetivos esenciales de la programación trataba de introducir el discurso de la energía en la agenda del proceso de recuperación. Y se promovió la construcción de un discurso urbano de la energía a diferentes escalas que sucintamente refiero a continuación. 


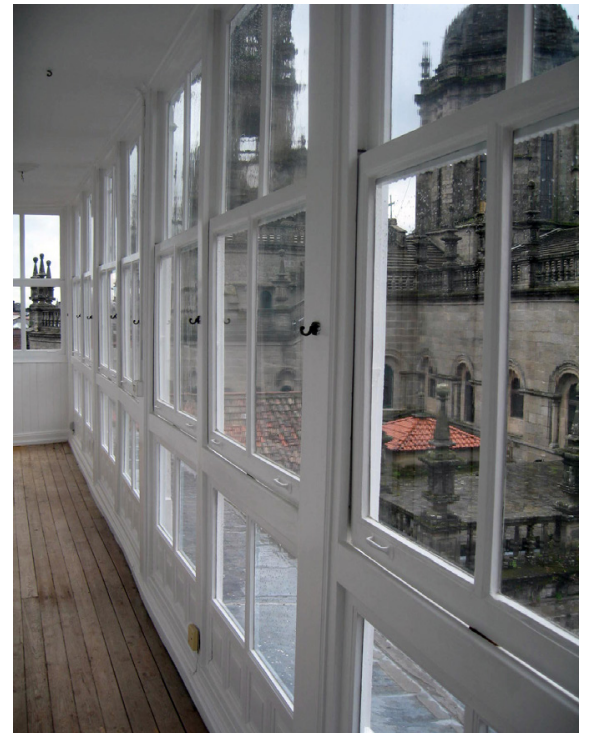

La aproximación desde la energía al patrimonio histórico urbano permitió sustituir el catálogo tradicional de elementos arquitectónicos seleccionados con criterios histórico-artísticos que, por defecto, se suele acabar gestionando con criterios ambientalistas, por un catálogo en base al valor de los elementos arquitectónicos para la gestión higrotérmica de los edificios, que se puede gestionar fácilmente con criterios de habitabilidad y uso. Al final, ambos catálogos terminan siendo muy similares, pero hablando de habitabilidad y uso es más fácil entenderse con los habitantes. Los muros de mampostería medievales se convierten en acumuladores de energía y las galerías en colectores solares | foto Adrián Martín Prieto
En primer lugar y con carácter básico, se trabajó en una revisión crítica de las características físico-constructivas de las arquitecturas históricas, en la que el análisis y valoración de estas arquitecturas trata de desprenderse del ensimismamiento característico de la visión patrimonialista para construir un argumentario basado en la comprensión de su funcionamiento higrotérmico y constructivo. Con ese objetivo se impulsó el denominado Laboratorio Urbano de la Energía, se participó en el proyecto Europeo Energy Efficiency for EU Historic District's Sustainability (EFFESUS) y se estableció un convenio de colaboración con el Departamento de Energía y Medio Ambiente de la Architectural Association School of Architecture.

Esta nueva forma de aproximarse al patrimonio urbano permitió descartar el catálogo tradicional de elementos arquitectónicos a proteger por su valor histórico, para comenzar a hablar en su lugar de un catálogo de elementos arquitectónicos con valor para la gestión higrotérmica de los edificios y viviendas. Suena radical, pero se pudo comprobar que, al final, ambos catálogos terminan siendo bastante similares. Los muros de mampostería medievales se convierten en acumuladores de energía y las galerías en colectores solares, pero con la ventaja de que es más fácil entenderse con los ciudadanos hablando de energía que del valor histórico abstracto de cualquier elemento arquitectónico. Así, por ejemplo, la madera ya no es un material a conservar por ser tradicional, sino porque es uno de los raros materiales de construcción que se producen con energía solar, y, en lugar de emitir gases de efecto invernadero en su producción, los captura.

\section{MANTENIMIENTO VERSUS REHABILITACIÓN}

El trabajo realizado desde la Oficina Técnica del Consorcio de Santiago durante los últimos años del siglo XX en la ciudad histórica conquistó la confianza de los ciudadanos demostrando que la rehabilitación en términos de mejora de habitabilidad y condiciones de vida era posible. El discurso de la energía y esa cultura de la rehabilitación consolidada en Santiago de Compostela permitió establecer a partir de 2006 un diálogo colaborativo entre la administración y los ciudadanos en el que, en lugar de rehabilitación, se prefirió comenzar a hablar de habitabilidad. En ese contexto y convencidos de que es el mantenimiento, mucho más que la rehabilitación, la clave de la conservación del patrimonio urbano, se lanzaron los programas de mantenimiento Ter é manter (Tener es mantener), destinado a edificios y viviendas, y A Pedra que Pisas (La piedra que pisas), dirigido al mantenimiento de los pavimentos históricos de Compostela.

El ritmo natural de la transformación de la ciudad histórica tiene más que ver con el mantenimiento que con la rehabilitación. El abandono de la cultura del mantenimiento contribuyó significativamente al progresivo deterioro de 


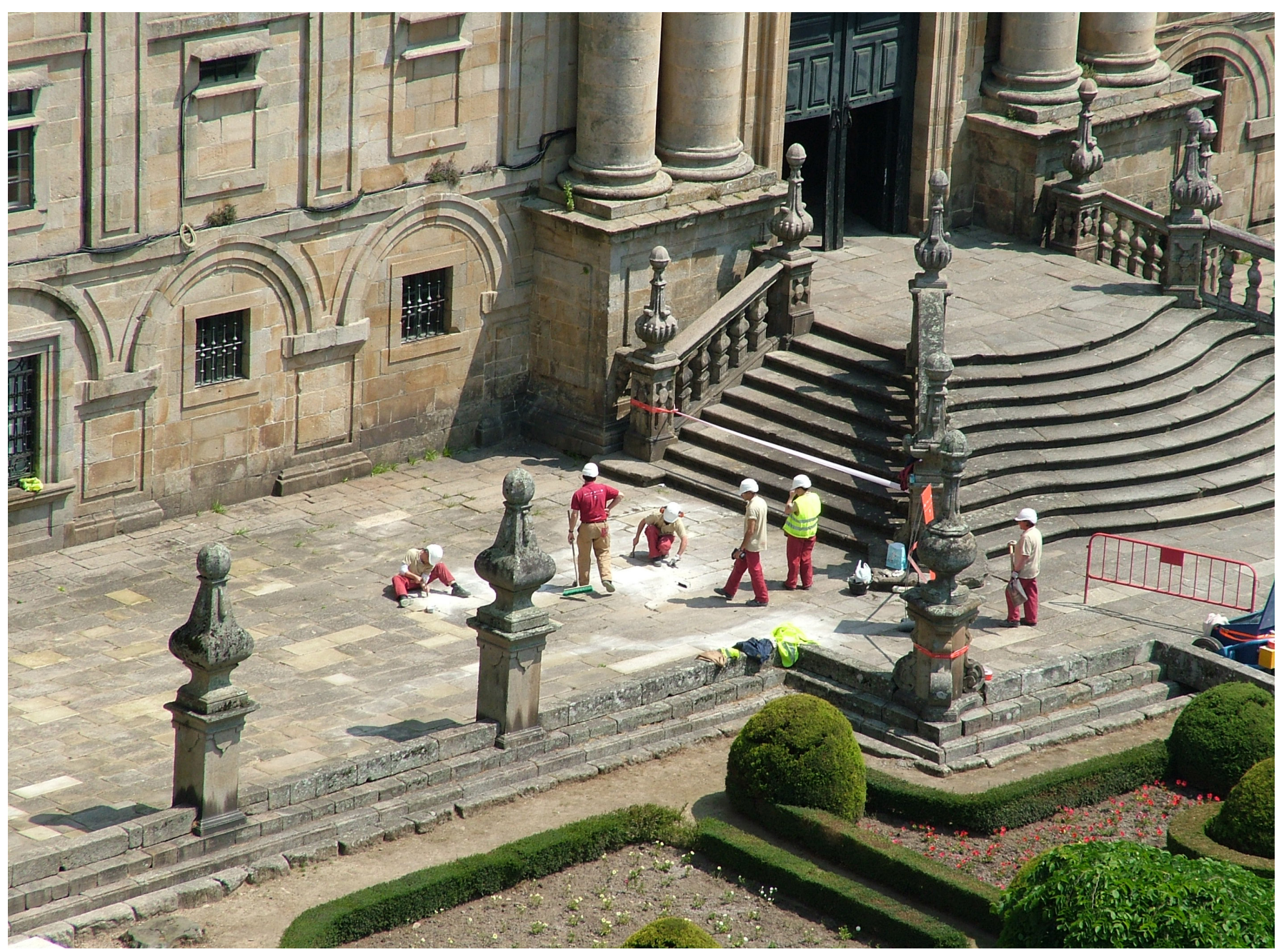

La cultura del mantenimiento, mucho más que la rehabilitación, es la clave de la conservación del patrimonio urbano. El programa "A Pedra

las arquitecturas de la ciudad histórica y está directamente relacionado con el proceso de colapso constructivo de los centros históricos. Se trata de un colapso coherente con el predominio de la "ciudad de la renta" sobre la "ciudad de la reproducción social", pues la expectativa de renta no solo se alique Pisas", aún hoy vigente, se puso en marcha en el año 2007 para impulsar una dinámica de mantenimiento constante de los $60.000 \mathrm{~m}^{2}$ de enlosados de granito del pavimento de la ciudad histórica | Adrián Martín Prieto menta con el progresivo colapso físico y el desalojo de habitantes, sino que necesita por definición el deterioro irreversible del centro histórico para materializarse como alternativa. El avanzado estado de abandono y decaimiento de muchas ciudades históricas nos acostumbró a pensar en términos de rehabilitación, restauración o reestructuración a la hora de programar estrategias de intervención, pero el verdadero soporte de su realidad constructiva es y ha sido la cultura del mantenimiento. El mantenimiento es naturalmente también una razón elemental de sostenibilidad y eficiencia en la administración de recursos, pero, sobre todo, la normalización de la cultura y hábito del mantenimiento en los centros históricos permite garantizar su conservación 
El programa "Ter é Manter" (Tener es mantener) fue el primer programa de subvenciones a mantenimiento desarrollado en un centro histórico Patrimonio de la Humanidad. Su primera convocatoria se hizo en el año 2006 con el objetivo de recuperar el hábito del mantenimiento de

edificios y viviendas. Hoy en día sigue vigente bajo

la dirección de la arquitecta Lourdes Pérez Castro | fuente Cenlitrosmetrocadrado y Consorcio de Santiago

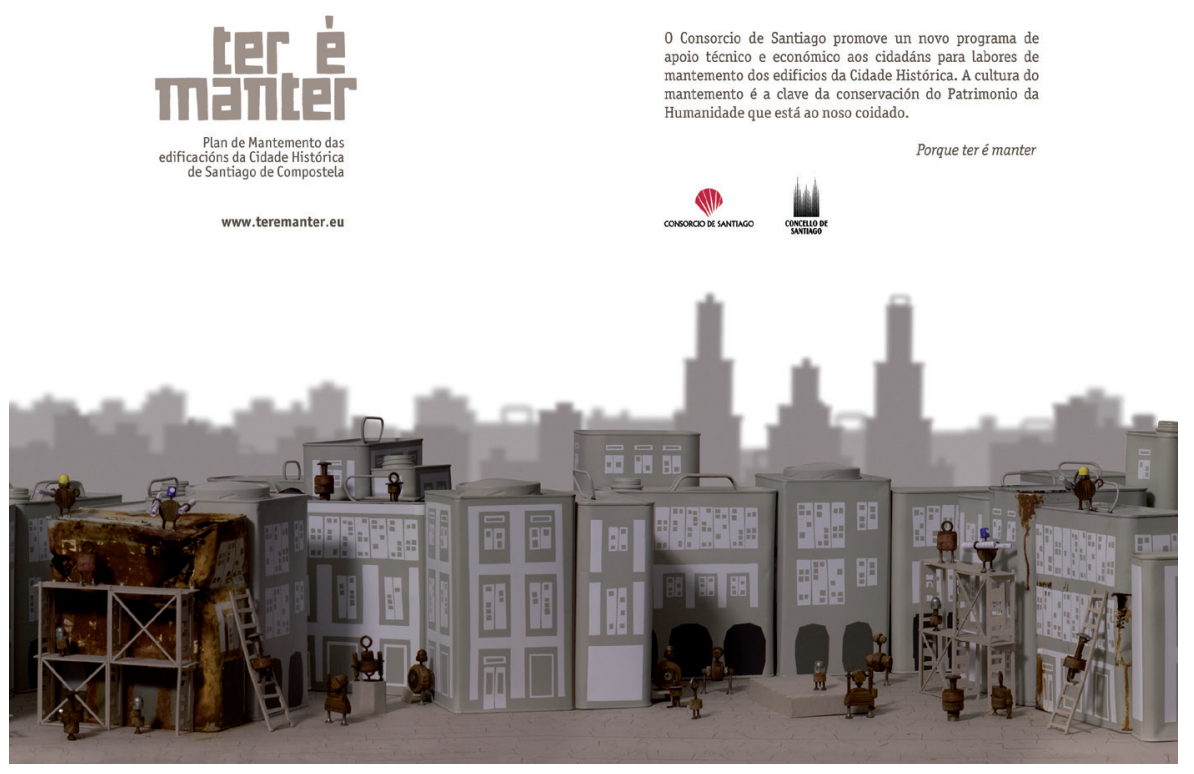

creando empleo local especializado y estable. Además, como se acreditó en los estudios previos realizados en Santiago, la inversión en mantenimiento preventivo es más rentable y genera ya, en el medio plazo, ahorro significativo, público y privado, en el gasto de conservación del patrimonio urbano.

\section{EL PLAN DIRECTOR DE INFRAESTRUCTURAS}

Esta propuesta de transformación en la forma de entender la conservación y puesta en valor de una ciudad Patrimonio de la Humanidad fue posible porque la denominada rehabilitación pacífica de los edificios y viviendas de la ciudad histórica estaba en el año 2006 socialmente asumida, incluso fuera de los programas oficiales de ayudas. Este era un factor de éxito de las políticas desarrolladas, pero muy pronto puso en evidencia que el punto débil de la habitabilidad de los edificios de la ciudad histórica estaba en la red urbana de infraestructuras y suministros. En efecto, la red de infraestructuras y suministros de la ciudad histórica, desplegada en su mayor parte con cierta improvisación durante el siglo XX, se puede considerar obsoleta, ineficiente e inadaptada a las exigencias contemporáneas de habitabilidad. Incluso independientemente del estado de ruina técnica de estas infraestructuras, no es solamente una cuestión de habitabilidad o eficiencia energética, sino, sobre todo, de competitividad y productividad de las viviendas, oficinas o locales comerciales de la ciudad histórica. Creíamos entonces, y seguimos creyendo hoy, que el plan director de infraestructuras es indispensable para garantizar la vigencia habitacional de la ciudad histórica, para garantizar su eficiencia energética y, en definitiva, para garantizar su viabilidad como espacio de convivencia. 


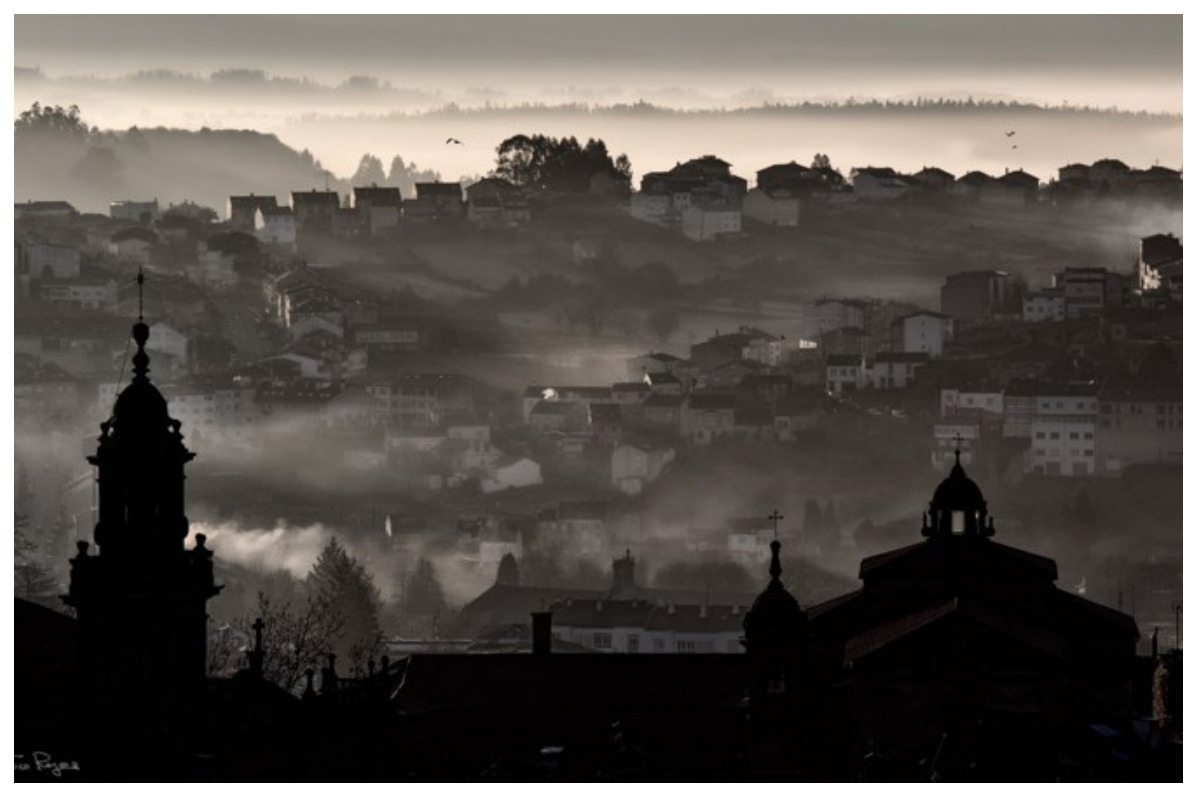

La hoja de ruta para impulsar el Plan Director de Infraestructuras de la ciudad histórica de Santiago se propuso en el año 2009, con un horizonte de desarrollo ejecutivo de 10 años y una inversión total superior a los 110 millones de euros. El plan se apoya en la puesta en valor del dominio público subterráneo y contempla la incorporación del suministro de energía, district heating, a todos los edificios del área Patrimonio de la Humanidad.

En realidad hay que advertir que la eliminación del cableado aéreo es una vieja aspiración en relación con la apariencia de la ciudad histórica, pero cuando se estudia la verdadera trascendencia de la modernización del equipamiento de infraestructuras que es necesario realizar urgentemente en las ciudades históricas por razones estrictas de habitabilidad, competitividad y productividad, el mandato de eliminación de cableado aéreo por razones ambientalistas vuelve a sonar francamente intrascendente. El Plan Director de Infraestructuras Urbanas se entendía complementario de un Plan inteligente de Logística y Gestión de Mercancías. Ambos estaban destinados tanto a multiplicar la competitividad de las empresas que optasen por radicarse en la ciudad histórica como a resolver problemas cotidianos de los habitantes dando soporte al proceso de peatonalización indispensable para recuperar el espacio público.

\section{ESTRATEGIA DE VERDE URBANO Y GESTIÓN DEL AGUA}

La estrategia de rehabilitación urbana sostenida en Santiago durante los últimos 30 años supuso la incorporación a dominio público a través del Consorcio de Santiago de miles de metros cuadrados de huertas y otros
Con las dinámicas de rehabilitación en marcha, se hizo evidente que la habitabilidad de la ciudad histórica de Santiago dependía de las infraestructuras urbanas. La subterraneización de los cableados muy lejos de tener que ver con la apariencia, es indispensable por razones de habitabilidad, competitividad y productividad urbana. El Consorcio de Santiago formuló en el año 2010 un Plan para la renovación de infraestructuras urbanas, que incluía el suministro de energía. La estrategia verde, el Plan de Infraestructuras y el Plan de logística y gestión de mercancías estaban llamados a ser la locomotora de la transformación urbana de Compostela | foto Fuco Reyes 
La protección en el Plan General de 1989 de huertas y otros vacíos urbanos y su incorporación a dominio público promovida por el Consorcio de Santiago desde el año 1994, representa una oportunidad excepcional de impulsar programas de estímulo de la economía local, agricultura urbana, gestión de las consecuencias del Cambio Climático, salud pública, gestión del agua y movilidad sostenible. Con ese objetivo el Consorcio de Santiago impulsó en el año 2008 en colaboración con la UPC y bajo la dirección de Albert Cuchí "La estrategia verde de Compostela" | fuente Consorcio de Santiago vacíos urbanos. Ese patrimonio público de vacíos urbanos representaba ya en el año 2008 una oportunidad única de incorporar al proceso de recuperación urbana de Compostela una gran variedad de programas de estímulo de la economía local, de agricultura urbana, de salud pública o de movilidad sostenible.

Y con ese objetivo se impulsó la construcción de un discurso del agua y del verde urbano en confluencia con el Plan Director de Infraestructuras y el Plan de logística y gestión de mercancías. "La estrategia verde de Compostela" se promovió en colaboración con la Universidad Politécnica de Catalunya, bajo la dirección del profesor Albert Cuchí (Cuchí Burgos 2010), tratando de argumentar las posibilidades que ofrece un nuevo enfoque, caracterizado por la consideración de la sostenibilidad como un reto ineludible, como un instrumento transformador de nuestra sociedad y, con ella, de nuestras ciudades. El programa pretendía documentar y dar fundamento a la gran transformación de las políticas y objetivos de conservación del patrimonio urbano de Compostela que proponía el Consorcio de Santiago. Desde la energía, el medioambiente y la sostenibilidad, la conservación del patrimonio se enriquece con valores y argumentos que desbordan el concepto tradicional de conservación urbana. Y esto es así porque el patrimonio entonces deja de estar solo relacionado con la continuidad histórica, las raíces o la identidad,

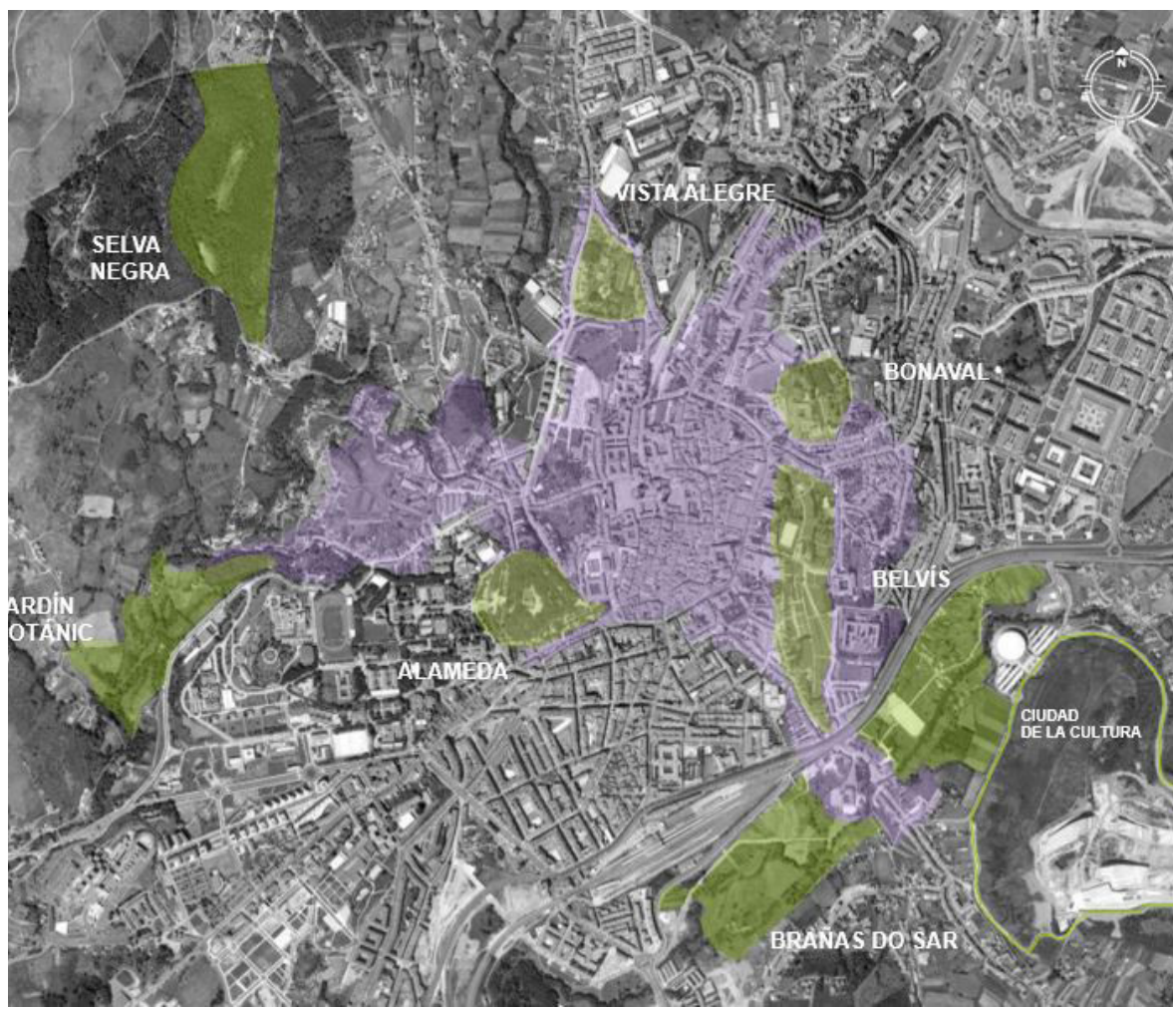




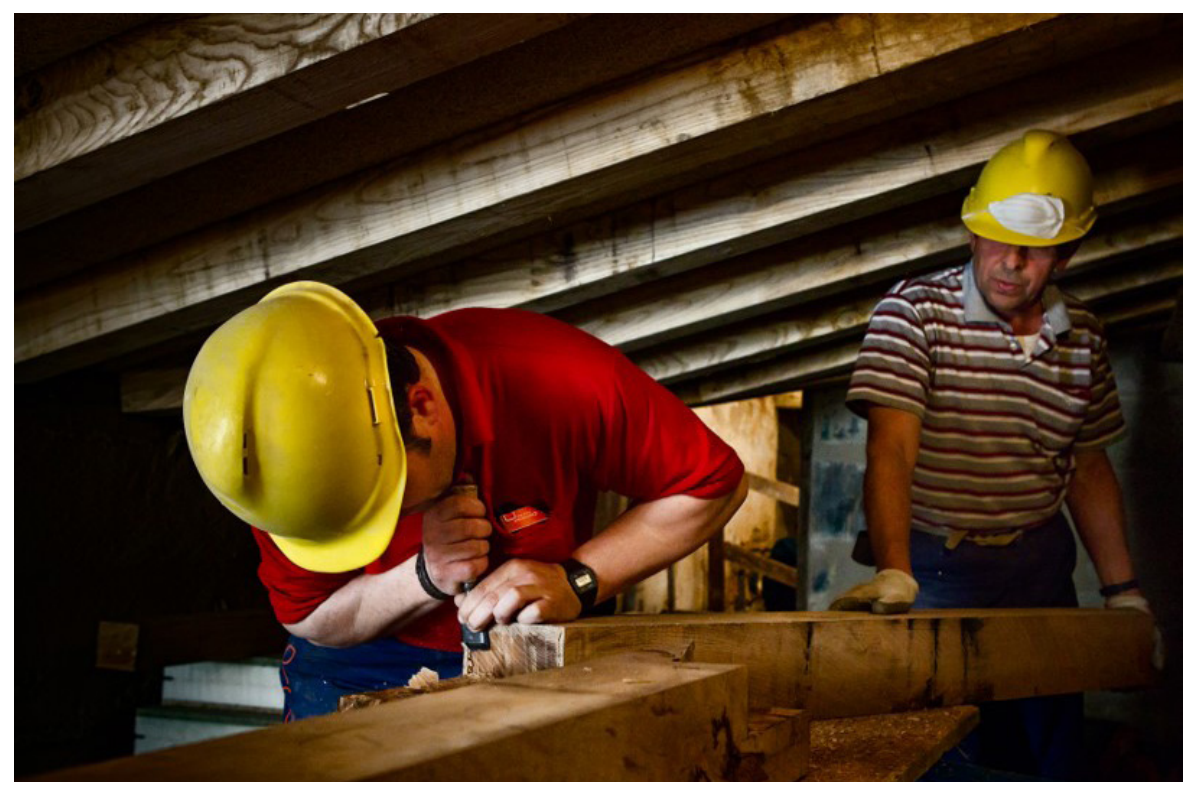

para incorporar valores que deben ser recuperados y salvaguardados porque encierran lecciones de gestión de recursos en el territorio que debemos recuperar y transmitir si queremos avanzar hacia la construcción de una sociedad ambientalmente sostenible. Y esa es la clave de rehabilitar para garantizar un mundo mejor.

\section{EPÍLOGO}

La formulación de estas iniciativas pretendía impulsar una nueva forma de gestionar la regeneración y revitalización de una ciudad histórica Patrimonio de la Humanidad, superando los modelos patrimonialistas que, francamente, más bien parecen agotados e incluso, en muchos casos, responsables de la pérdida de pulso ciudadano que tanto devalúa los centros históricos. Pero esta transformación en la forma de entender y manejar una ciudad Patrimonio de la Humanidad solo es posible en el marco de estas dos últimas condiciones generales:

La primera tiene que ver, a imagen de lo que sucede en relación con la salvaguarda del patrimonio natural, con la denominada custodia del territorio (Basora Roca y Sabaté y Rotés 2006), con el estímulo entre los ciudadanos de una dinámica de custodia de la ciudad histórica. La implicación ciudadana debe ser activa y protagonista de la gestión de la ciudad histórica. Necesitamos construir una estrategia de implicación de los ciudadanos que no se limite a la estricta participación. Para ello es indispensable la información, el conocimiento y la educación, que nos lleva a la segunda condición

El estímulo entre los ciudadanos de una dinámica de custodia activa de la ciudad histórica no debe limitarse a la estricta participación ciudadana o interpretación de la ciudad histórica. Es indispensable la información, el conocimiento y la educación activa, entendidas como herramientas de gestión y estímulo de economías locales, capaces de generar no solo oportunidades, sino empleo cualificado y estable | foto Fuco Reyes 


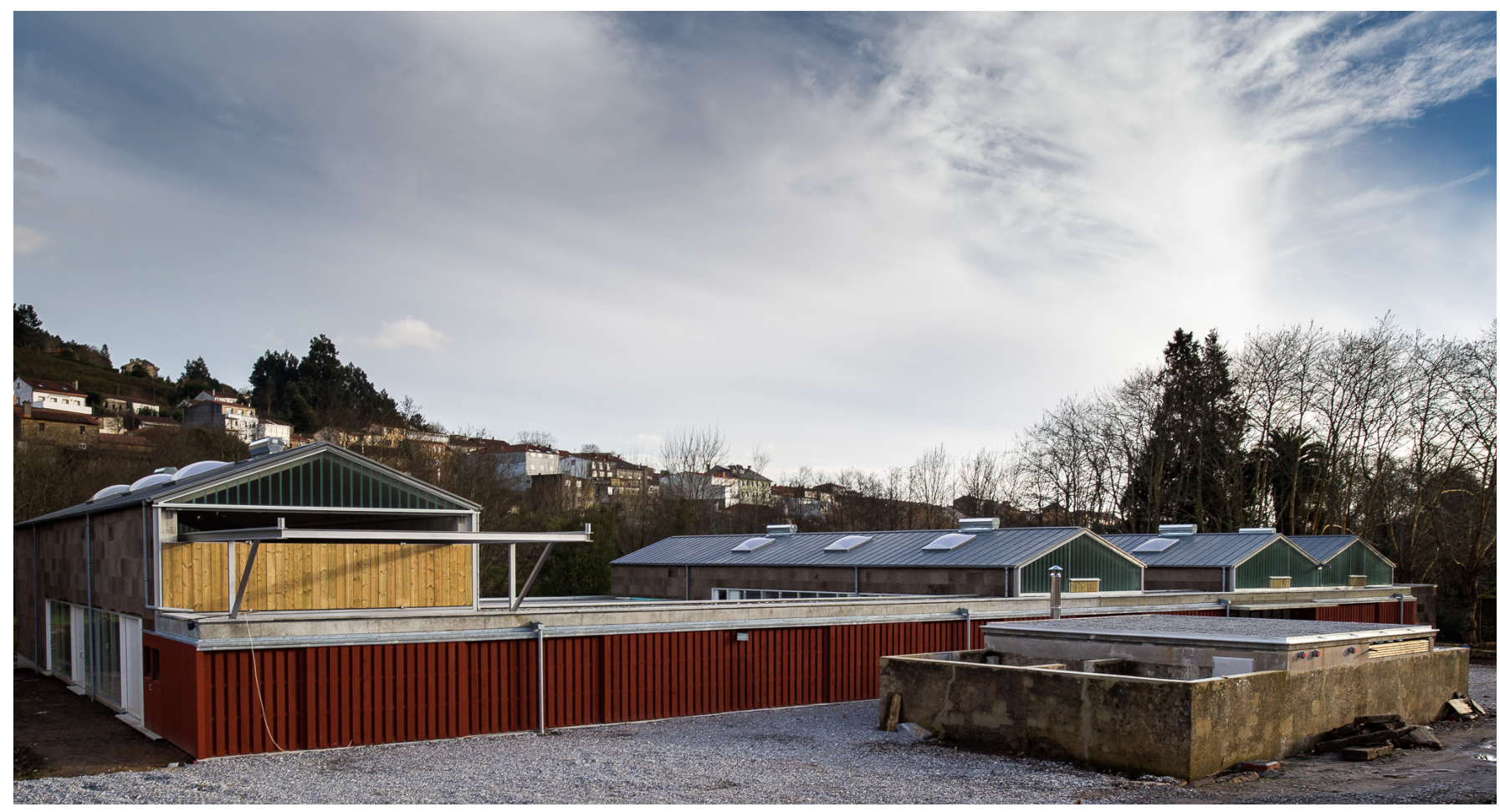

Con el objetivo de hacer de la rehabilitación urbana una oportunidad para el empleo, el Consorcio de Santiago y la Fundación Laboral de la Construcción impulsaron en el año 2008 la creación del Centro Nacional de referencia en formación en materia de rehabilitación en la antigua fábrica de curtidos de Pontepedriña de arriba. El Centro Nacional de referencia funciona desde el año 2018 a unos 2 $\mathrm{km}$ del la Plaza del Obradoiro en las instalaciones rehabilitadas de la antigua fábrica de curtidos, proyecto que mereció el Premio Galicia de Arquitectura y Urbanismo 2017-2018 | foto Fuco Reyes

general. Estoy convencido de que solo a medio plazo, de la mano de la educación, el conocimiento y la formación profesional, podremos avanzar con el objetivo declarado. Por eso el Consorcio de Santiago, en colaboración con la Fundación Laboral de la Construcción, impulsó el Centro Nacional de Formación en materia de rehabilitación de Santiago de Compostela que es hoy ya una realidad en las instalaciones rehabilitadas de una antigua fábrica de curtidos en la ribera del río Sarela.

Me gustaría terminar recopilando las citas de referencia que he utilizado para finalizar otros artículos o conferencias durante este tiempo. Empecé haciendo referencia hace 25 años, cuando estaba implicado principalmente con la intervención física desde la arquitectura y la construcción, a los versos de Isabel Escudero, "De la mano del aire", que era una declaración de intenciones y posición de partida como arquitecto a la hora de intervenir sobre lo construido. Más adelante y a medida que la experiencia de gestión integrada de la ciudad histórica crecía, terminaba optimista con la "ciudad histórica es un arma cargada de futuro", parafraseando los versos de Gabriel Celaya dedicados a la poesía. Hoy quiero terminar con una reflexión de Walter Benjamin que creo explica muy bien la dificultad colectiva para avanzar en la gestión del patrimonio urbano: "En cada época, se debe hacer un nuevo intento por arrancar a la tradición de los brazos del conformismo, que está siempre dispuesto a dominarla". 


\section{BIBLIOGRAFÍA}

- Álvarez Mora, A. (2016) Ciudad como producto versus la ciudad como obra. La realidad urbana entre el espacio de la renta y el espacio social. Lección inaugural curso 20152016. Valladolid: Ediciones Universidad de Valladolid

- Bases reguladoras do procedemento de edificios para a súa inclusión en programas de edificios tutelados (PET) Boletín Oficial de la Provincia de A Coruña, n. ${ }^{\circ} 297,27$ de diciembre de 2008, pp. 15249-15253. Disponible en: https://bop.dicoruna.es/bopportal/publicado/2008/12/27/ Bop20081227.pdf [Consulta: 29/06/2021]

- Basora Roca, X. y Sabaté y Rotés, X., (2006) Custodia del territorio en la práctica. Manual de introducción a una nueva estrategia participativa de conservación de la naturaleza y el paisaje. Fundació Territori i Paisatge. Disponible en: https://custodia-territorio.es/manual-decustodia-del-territorio-en-la-practica [Consulta: 14/06/2021]

- Campos Venuti, G. (1981) Urbanismo y austeridad. Madrid: Siglo XXI Editores

- Cuchí Burgos, A. (2010) Una estrategia verde para Santiago. Santiago de Compostela: Consorcio de Santiago. Disponible en: http://www.consorciodesantiago.org/es/unaestrategia-verde-para-santiago [Consulta:14/06/2021]

- Panero Pardo, A. (coord.) (2012) Programa de Edificios Tutelados, rúa do Medio, 65. Santiago de Compostela: Consorcio de Santiago (Cuadernos Técnicos). Disponible en: http://www.consorciodesantiago.org/sites/default/files/ 20120322_tutelados_rua_do_medio_small.pdf [Consulta:

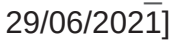

- Ramos Guallart, J., Panero Pardo, A., Camiruaga Osés, I., Tomé Ferreiro, P. y Fernández Hermida, R. (2002a) La arquitectura histórica y los criterios de rehabilitación. La rehabilitación de Santiago. La ciudad histórica de Santiago de Compostela, soporte de la vivienda del siglo XXI. Santiago de Compostela: Consorcio de Santiago. Disponible en: http:// www.consorciodesantiago.org/sites/default/files/tiii_los_ criterios_espanol.pdf [Consulta: 29/06/2021]

- Ramos Guallart, J., Panero Pardo, A., Camiruaga Osés, I., Tomé Ferreiro, P. y Fernández Hermida, R. (2002b) La gestión de la rehabilitación. La rehabilitación de Santiago. La ciudad histórica de Santiago de Compostela, soporte de la vivienda del siglo XXI. Santiago de Compostela: Consorcio de Santiago. Disponible en: http://www. consorciodesantiago.org/sites/default/files/tii_la_gestion_ espanol.pdf [Consulta: 29/06/2021]

- Rueda Palenzuela, S, (coord.) (2018) Carta para el diseño de nuevos desarrollos urbanos y la regeneración de los existentes. Agencia de Ecología Urbana de Barcelona. Disponible en: https://cartabcnecologia.files.wordpress. com/2018/05/carta-para-el-disec3b1o-de-nuevosdesarrollos-urbanos.pdf [Consulta: 26/08/2021]
- Unesco (2011) Recomendación sobre el paisaje urbano histórico, con inclusión de un glosario de definiciones, 10 de noviembre de 2011. Disponible en: http://portal.unesco.org/ es/ev.php-URL_ID=48857\&URL_DO=DO_TOPIC\&URL_ SECTION=201.httml [Consulta:14/06/2021]

- Verdú Martínez, M.T. (2018) Herramientas en relación con la vivienda vacía en España. Ciudady Territorio, vol. L, n. ${ }^{\circ} 197$, pp. 577-608. Estudios comparados. Disponible en: https:// recyt.fecyt.es/index.php/CyTET/article/view/76683/46999 [Consulta: 27/08/2021] 